\title{
PIGMENTS CAROTÉNOIDES ET REPRODUCTION DES POISSONS
}

\author{
G. CHOUBERT
}

I.N.R.A. - Laboratoire de Nutrition des poissons, Station d'Hydrobiologie,

Saint-Pée-sur-Nivelle - 64310 ASCAIN (France)

\begin{abstract}
RESUME
Cette revue bibliographique a pour but de préciser le devenir et le rôle des pigments caroténoïdes chez le poisson au cours des processus de la reproduction pris au sens large : âge à la première reproduction, mobilisation des pigments, maturation des oocytes, fécondation des ovules, embryogénèse.
\end{abstract}

\section{CAROTENOID PIGMENTS AND REPRODUCTION OF FISH}

\section{SUMMARY}

This bibliographical review intends to determine the development and role of carotenoid pigments in fish during their reproduction cycle: age at first reproduction, pigment mobilization. oocytes maturation, egg fertilization, embryogenesis.

\section{I - INTRODUCTION}

Les caroténoïdes sont des pigments liposolubles, colorés en jaune orangé ou rouge, que l'on rencontre dans de nombreux tissus végétaux. Ils sont susceptibles d'être métabolisés et stockés par l'organisme animal.

Si leur rôle dans la pigmentation des poissons ne fait plus aucun doute (CHOUBERT, 1981), il ne s'agit là que d'un aspect particulier de leurs fonctions car leur intervention a été notée dans des phénomènes physiologiques tels que celui de la croissance (DEUFEL, 1965) mais aussi celui de la reproduction (HARTMAN et al., 1947; STEVEN, 1948; DEUFEL, 1965). Le mode d'action de ce groupe chimique de pigments n'est pas connu, mais il semble qu'il soit lié à leur caractère provitaminique $A$ (GOODWIN, 1952).

Dans ce qui suit nous n'aborderons que les relations existantes entre les caroténoides et la reproduction des poissons, renvoyant le lecteur, pour les autres aspects du métabolisme des caroténoïdes, aux ouvrages de GOODWIN (1952), FOX (1953), ISLER (1971) et CHOUBERT (1981).

\section{2 - NATURE DES PIGMENTS CAROTENOIDES DES GONADES DE POISSONS}

\section{1. Caractères chímiques généraux des caroténoïdes}

Les caroténoïdes sont des systèmes polyéniques plus ou moins conjugués et leurs structures résultent d'un arrangement d'unités isopréniques autour d'une double liaison centrale *.

La formule éclatée d'une molécule d'astaxanthine, par exemple, révèle les grands traits de sa complexité (figure 1): deux cycles $\beta$-ionones aux extrémités; une chaîne de doubles liaisons conjuguées pouvant s'isomériser spontanément sous l'action de la lumière, d'enzymes ou de la chaleur et transformant ainsi l'allure de la molécule; des groupes fonctionnels pouvant se transformer par voies enzymatiques ou chimiques.

A l'évidence, une molécule possédant une structure si variée pourra participer à un grand nombre de réactions biochimiques sous l'influence de facteurs tant endogènes qu'exogènes.

* On trouvera dans les monographies de KARRER et JUCKER (1950), ZECHMEISTER (1962) et ISLER (1971) des développements sur la chimie des caroténoïdes. 
<smiles>CC1=C(/C=C/C=C/C=C/C(C)=C/C=C/C(C)=C/C=C/C=C(C)/C=C/C2=C(C)CCCC2(C)C)C(C)(C)CCC1</smiles>

\section{B-carotène \\ $(\beta-\beta$-carotène $)$}<smiles>CC1=C(/C=C/C(C)=C/C=C/C(C)=C/C=C/C=C(C)/C=C/C=C/C=C/C=C/C(C)=C/C=C/C2C(C)C(=O)CCC2(C)C)C(C)(C)CCC1=O</smiles>

\section{Canthaxanthine}

$(\beta-\beta$-carotène-4,4'-diol.)

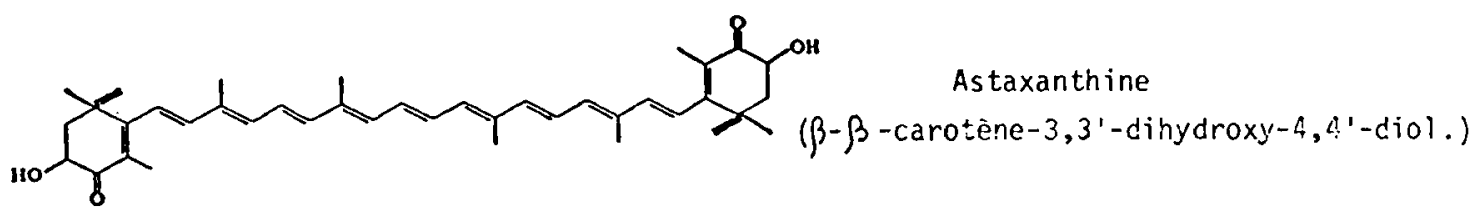<smiles>CC(C=CC=C(C)C=CC1=C(C)CC(O)CC1(C)C)=CC=CC=C(C)C=CC(C)=CC=CC=C(C)C=CC1C(C)CC(O)CC1(C)C</smiles><smiles>CC1=C[C@@H](O)CC(C)(C)[C@H]1/C=C/C(C)=C/C=C/C(C)=C/C=C/C=C(C)/C=C/C=C(C)/C=C/C1=C(C)CC(O)CC1(C)C</smiles>

Figure 1: Structure de quelques pigments caroténoïdes identifiés dans les gonades de poissons.

Figure 1: Structures of important carotenoids identified in fish gonads.

\subsection{Aspect qualitatif}

Les premières recherches sur la nature des pigments caroténoïdes ont été limitées par les moyens d'investigation et de caractérisation de ces pigments. Les études sur ces composés étaient basées sur des observations spectrales suivies quelquefois d'essai de partage des pigments entre différents solvants. C'est la raison pour laquelle, dans la plupart des analyses effectuées, seuls les composés majeurs ont été identifiés. Aujourd'hui, les techniques d'analyses tant physiques que chimiques, souvent à l'échelle de la microquantité, permettent l'identification de pigments mineurs.

Les pigments caroténoïdes ont été signalés dans les ovaires, les ovules et les œufs des femelles (LEDERER, 1935 ; GOODWIN, 1952 ; CZECZUGA, 1975, 1979a) mais également dans les testicules et la laitance des mâles (STEVEN, 1948; GOODWIN, 1952; CZECZUGA, 1974).

D'une façon générale, les poissons d'eau douce ou côtiers ont des compositions en pigments caroténoïdes plus complexes que celles des poissons marins (HIRAO, 1967) (Tableau 1).

Nous me mentionnerons que quelques-uns des principaux pigments caroténoïdes mis en évidence dans les gonades de poissons:

- $\beta$-carotène: ce pigment fait partie des substances naturelles les plus universellement répandues tant chez les animaux que chez les végétaux (LEDERER, 1934). La structure de ce pigment présente deux cycles $\beta$-ionones terminaux seuls. C'est un précurseur de la vitamine $A$.

- Canthaxanthine: identifiée par HAXO (1950), ce pigment se caractérise par deux groupements cétones en position 4 et $4^{\prime}$. Aujourd'hui synthétisé artificiellement, il est également utilisé en pisciculture intensive pour la pigmentation de la chair.

- Astaxanthine : isolée dès 1876, sa structure chimique ne fut mise en évidence qu'en 1938 par KUHN \& SORENSEN. Ce pigment peut se trouver sous forme libre ou sous forme estérifiée (mono ou diester). 


\begin{tabular}{|c|c|c|c|}
\hline $\begin{array}{l}\text { Famille } \\
\text { Fanily }\end{array}$ & $\begin{array}{l}\text { Espèce } \\
\text { Species }\end{array}$ & $\begin{array}{l}\text { Tissus } \\
\text { Tissue }\end{array}$ & $\begin{array}{l}\text { Pigment caroténoîde } \\
\text { Carotenoid pigment }\end{array}$ \\
\hline Ammodytidae & Amodytes tobianus & Ovaire & $\beta$-carotène \\
\hline \multirow[t]{2}{*}{ Clupeidae } & Clupea harengus & Oeuf & Lutéine, canthaxanthine. astaxanthine \\
\hline & & Laitance & Isozéaxanthine, canthoxanthine, astaxanthine \\
\hline \multirow[t]{3}{*}{ Corregonidae } & Corregonus albula & Ovaire & Astaxanthine \\
\hline & & Oeuf & Astaxanthine \\
\hline & & Laitance & Zéaxanthine, canthaxanthine, astaxanthine \\
\hline Cyprinidae & Cyprinus carpio & Oeuf & $\beta$-carotène, canthaxanthine, astaxanthine \\
\hline \multirow[t]{3}{*}{ Gadidae } & Eleginus novaca & Ovaire & Non identifié \\
\hline & Gadus morhua & Oeuf & $\beta$-carotène, lutéine, astaxanthine \\
\hline & Lota lota & Deuf & Lutéine \\
\hline \multirow[t]{2}{*}{ Pleuronectidae } & Hippoglos sus hippoglossus & Ovaire & P-carotène, lutẽine, astaxanthine, \\
\hline & & Oeuf & $\boldsymbol{\beta}$-carotène, lutéine, astaxanthine \\
\hline \multirow[t]{6}{*}{ Salmonidae } & Saimo salar & Oeuf & B-carotène, zéaxanthine, astaxanthine \\
\hline & Oncorbunchus_keta & Oeuf & Canthaxanthine, as taxanthine \\
\hline & Salme gairdneri & $\begin{array}{l}\text { Oeuf } \\
\text { Laitance }\end{array}$ & $\begin{array}{l}\text { B-carotène } \\
\text { Zéaxanthine, astaxanthine }\end{array}$ \\
\hline & Salmo trutta & Deuf & $\beta$-carotène, lutéine, astaxanthine \\
\hline & Salmo irideus & Oeuf & Lutéine, astaxanthine \\
\hline & & Laitance & $\beta$-carotène, lutèine, canthaxanthine, astaxanthine \\
\hline Soleidae & Solea vulgaris & Oeuf & B-carotène \\
\hline Sparidae & Chrysoghrys maior & Ceuf & Canthaxanthine, astaxanthine \\
\hline
\end{tabular}

Tableau 1: Nature des pigments caroténoïdes des gonades de poissons d'après GOODWIN (T952). FOX (1957). CZECZUGA (1979), SIMPSON et al. (1981).

Table 1: Carotenoid pigment of ovaries and ova of fish. After GOODWIN (1952), FOX (1957). CZECZUGA (1979), SIMPSON et al. (1981).

- Zéaxanthine : isolée en 1929, sa structure ne fut élucidée que plus tard (KARRER \& JUCKER. 1950). Ce pigment est caractérisé par la présence de deux groupes hydroxyles en position 3 et $3^{\prime}$. II est peu représenté dans les gonades de poissons.

- Lutéine: ce pigment caroténoïde possède une structure de type $\alpha$-carotène (KARRER \& JUCKER, 1950). Ce pigment est assez fréquemment identifié chez le poisson. II peut se trouver sous forme libre ou estérifiée.

D'autres pigments caroténoïdes sont plus rarement détectés et généralement en quantités réduites. Ils représentent aussi pour certains d'entre eux des intermédiaires importants dans le métabolisme pigmentaire (LENEL et al., 1978). On peut d'ailleurs admettre que leur faible concentration ou même leur absence apparente est due. dans certains cas, à la rapidité de leur transformation (GILCHRIST \& GREEN, 1960).

Les pigments caroténoïdes se retrouvent dans les gonades, dissous dans la fraction lipidique. La forme sous laquelle les pigments caroténoïdes se présentent dans les gonades est encore controversée. Pour certains auteurs les xanthophylles seraient sous forme libre (HARTMAN et al., 1947; GOODWIN, 1952 ; FOX, 1957) pour d'autres elles seraient estérifiées (MIKI et al., 1982).

\subsection{Aspect quantitatif}

Les quantités de pigments caroténoïdes présents dans les gonades de même que les proportions relatives des différentes formes de caroténoïdes varient avec l'individu, l'âge. le sexe et l'époque de l'année (TSUKUDA \& AMANO, 1966; HIRAO, 1967; HERRING, 1969).

A titre d'exemple, le Tableau ll donne quelques valeurs de teneurs en pigments caroténoïdes des gonades de poissons.

\section{3 - ORIGINE DES PIGMENTS CAROTENOIDES DES GONADES DE POISSONS}

Les pigments caroténoïdes des gonades de poisson sont d'origine alimentaire. On sait, en effet, que les vertébrés en général et les poissons en particulier ne peuvent synthétiser les pigments caroténoides "de novo" et de ce fait doivent les trouver dans leur alimentation. (ANDRE, 1926). 
Bull. Fr. Pêche Piscic. (1986) $300: 25-32-28-$

\begin{tabular}{|c|c|c|c|c|c|}
\hline \multirow{3}{*}{$\begin{array}{l}\text { Famille } \\
\text { Family } \\
\text { Ammodytidae }\end{array}$} & \multirow{2}{*}{$\begin{array}{c}\text { Espèce } \\
\text { Species } \\
\text { Ammodytes tobianus }\end{array}$} & \multirow{2}{*}{$\begin{array}{l}\text { Tissus } \\
\text { Tissue }\end{array}$} & \multicolumn{2}{|c|}{$\begin{array}{l}\text { Quantitẽ et mode } \\
\text { d'expression } \\
\text { Quantity }\end{array}$} & \multirow{2}{*}{$\begin{array}{c}\begin{array}{c}\text { Rêférence } \\
\text { Reference }\end{array} \\
\text { G00DWIN, } 1952\end{array}$} \\
\hline & & & 0,83 & $\mathrm{mg} / 100 \mathrm{~g} \mathrm{sec}$ & \\
\hline & & Laitance & trace & & GOOOWIN, 1952 \\
\hline Carangidae & Seriola quinqueradiata & Ovaire & 0,23 & $\mathrm{mg} / 100 \mathrm{~g}$ & MIKI et al., 1984 \\
\hline \multirow[t]{2}{*}{ Cyprinidae } & Bartus barbus & ovaire & 1,20 & $\mathrm{mg} / 100 \mathrm{~g}$ & SHNAREVICH \& SAKHNENKO, 1971 \\
\hline & Leuciscus cephalus & Ovaire & 0,99 & $\mathrm{mg} / 100 \mathrm{~g}$ & SHNAREVICH SAKHNENKO, 1971 \\
\hline Gadidae & Gadus morrhua & Ovaire & 0,40 & $\mathrm{mg} / 100 \mathrm{~g}$ & MiKI et al., 1984 \\
\hline Percidae & Perca fluviatilis & Ovaire & 0,98 & $\mathrm{mg} / 100 \mathrm{~g}$ & SHNAREVICH \& SAKHNENKO, 1971 \\
\hline \multirow[t]{10}{*}{ Salmonidae } & Oncorhynchus nerka & Ovaire & 2,95 & $\mathrm{mg} / 100 \mathrm{~g}$ & CROZIER, 1969 \\
\hline & & Oeuf & 0,68 & $\mathrm{mg} / 100 \mathrm{~g}$ & KITAKARA, 1984 \\
\hline & Oncorhynchus keta & Deuf & 2,78 & $\mu g / g$ frais & CZECZUGA, 1979 a \\
\hline & Salmo trutta & Deuf & 1,74 & $\mu g / g$ frais & STEVEN, 1948 \\
\hline & & Oeuf & 1,27 & $\mu g / g$ frais & CZECZUGA, 1975 \\
\hline & & Oeuf & 2,87 & $\mu g / g$ frais & CZECZUGA, 19790 \\
\hline & & Ovaire & 1,50 & $\mathrm{mg} / 100 \mathrm{~g}$ & SHNAREVICH \& SAKHNENKO, 197 \\
\hline & & Laitance & 0,03 & $\mu g / g$ frais & CZECZUGA, $1979 \mathrm{~b}$ \\
\hline & Salmo irideus & Oeuf & 73,2 & $\mu g / g$ frais & HARTMAN et al., 1947 \\
\hline & Salmo gairdneri & Laitance & 0,24 & $\mu g / g$ frais & CZECZUGA, 1974 \\
\hline Sparidae & Chrysophrys major & Oeuf & 80 & $\mu \mathrm{g} / 100 \mathrm{~g}$ & MIKI et al.. 1984 \\
\hline
\end{tabular}

Tableau II: Distribution quantitative des caroténoïdes dans les gonades de poissons.

Table II : Quantitative distribution of carotenoid in ovaries and ova of fish.

\section{4 - PARTICIPATION DES PIGMENTS CAROTENOIDES AUX PROCESSUS DE LA REPRODUCTION}

\subsection{Mobilisation des pigments}

En période de maturité sexuelle, les gonades accumulent les pigments caroténoïdes.

Cette accumulation s'effectue chez la femelle à partir de son alimentation mais également à partir du réservoir que constitue le muscle. Ainsi, la couleur des oocytes du médaka (Oryzias latipes) dépend-elle directement de la quantité de pigments caroténoïdes ingérée par la femelle (TAKEUCHI. 1960). De même, chez la daurade (Chrysophrys major). MIKI et al., (1984) ont montré que les pigments caroténoïdes ingérés étaient directement stockés dans les oocytes après hydrolyses. STEVEN (1949) note, chez la truite fario (Salmo trutta) une mobilisation quasi totale des pigments caroténoïdes musculaires et corrélativement un accroissement quantitatif important de ceux des ovaires. La quantité de pigments contenus dans le muscle semble toutefois insuffisante pour assurer la fourniture des pigments déposés dans les ovaires. Le poisson doit donc trouver un supplément dans la nourriture naturelle qu'il consomme durant la maturation des ovules. Au contraire, CROZIER (1969) a calculé que les ressources musculaires étaient largement excédentaires chez le saumon keta (Oncorhynchus keta), chez lequel 60 p. 100 des pigments caroténoïdes sont métabolisés au cours de la migration de reproduction.

Chez le mâle, si la mobilisation des pigments caroténoïdes vers les testicules et la laitance n'a pas été signalée, en revanche des transferts de pigments ont lieu vers la peau en période de fraie comme l'ont montré GOODWIN (1962) chez la poule de mer (Cyclopterus lumpus) et le fondule (Fundulus parvipinnis) et CROZIER (1969) chez le saumon keta. Cet accroissement de la pigmentation de la peau des mâles a été interprété comme jouant un rôle dans le comportement de reproduction: attraction des femelles (KRINSKY, 1971; MIKULIN \& SOIN, 1975).

\subsection{Maturation des oocytes}

Seul l'effet de la canthaxanthine sur la maturation des oocytes a été étudié.

Dans une expérience d'une durée de 6 mois, DEUFEL (1965) a nourri des truites arc-en-ciel (Salmo gairdneri) de 2 ans avec deux régimes ne se différenciant que par leur teneur en pigments caroténoïdes, le régime expérimental étant supplémenté avec $40 \mu \mathrm{g}$ de canthaxanthine par $\mathrm{kg}$ d'aliment. II a été observé des différences quant à la maturité sexuelle : $25 \mathrm{p} .100$ des femelles étaient matures chez les animaux témoins contre $64.5 \mathrm{p} .100 \mathrm{chez}$ les animaux ayant ingéré de la canthaxanthine. Des observations complémentaires ont été effectuées quant à la date de la fraie (plus précoce de 
10 jours chez les poissons expérimentaux) et quant au pourcentage d'œufs fécondés $(99,9 \mathrm{p}$. $100 \mathrm{chez}$ les poissons expérimentaux contre 95.7 p. 100 chez les poissons témoins. Ces résultats expérimentaux n'ont pu être confirmés jusqu'à l'heure.

MORRISON et SMITH (1981) ne notent pas de différence de fécondité chez des femelles de saumon de fontaine (Salvelinus fontinalis) nourries pendant deux mois avant la fraie d'un aliment enrichi en canthaxanthine. HARRIS (1984) ne rapporte pas non plus de différence de fécondité entre femelles de truite arc-en-ciel (Salmo gairdneri), nourries pendant 6 mois d'un régime contenant 20 mg de cathaxanthine par $\mathrm{kg}$ d'aliment, par rapport à des poissons témoins. Cét auteur note seulement que les cufs des truites témoins sont légèrement plus petits que ceux provenant de poissons ayant ingéré de la canthaxanthine.

\subsection{Fécondation des ovules}

DEUFEL (1965) et GEORGIEV (1971) chez la truite (Salmo gairdneri), WATANABE et al. (1984) chez la daurade (Chrysophrys major) ont noté que le taux de fécondation des ovules pigmentés était supérieur à celui d'ovules non pigmentés.

HARTMAN et al. (1947) ont émis l'hypothèse de l'intervention des pigments caroténoïdes dans les phénomènes de fécondation des ovules de poissons. Ainsi, le $\beta$-carotène, la lutéine et l'astaxanthine auraient un effet positif sur la mobilité des spermatozoïdes mais seule l'astaxanthine et le $\beta$-carotène, bien que ce dernier serait moins actif, induiraient une chémotaxie positive. Sur la base de l'effet antagoniste de la seule astaxanthine envers l'androgamonel, ces auteurs estiment que ce pigment caroténoïde jouerait le rôle d'hormone vraie.

Ce rôle est cependant mis en doute par MIKULIN et SOIN (1975) compte tenu du caractère fortement hydrophobe de cette molécule, ce qui semble être confirmé par OUANTZ(1980). Cet auteur ne note aucune différence significative dans la fécondation d'ovules de truites arc-en-ciel (Salmo gairdneri) nourries pendant 8 à 15 semaines avant la fraie de régimes alimentaires enrichis en canthaxanthine.

Il faut toutefois signaler que la fécondation des ovules de truites (Salmo gairdneri) peut varier dans des proportions très importantes selon la date de ponte, l'âge ou le poids du corps du poisson (ESCAFFRE et BILLARD, 1979) c'est-à-dire en dehors de toute considération pigmentaire.

\subsection{Respiration des cuts}

L'intervention des pigments caroténoïdes dans la respiration des cufs de poissons a été particulièrement développée par MIKULIN et SOIN (1975) en se basant sur les observations suivantes :

- il a été établi, par l'étude de poissons appartenant à des groupes écologiques différents, que les œuifs se développant dans des eaux peu oxygénées sont plus pigmentés que ceux se développant dans des eaux bien oxygénées (SOIN, 1962).

- il a été rapporté une corrélation entre le niveau de pigmentation des œufs et l'importance du système sanguin périphérique des vésicules vitellines qui constitue l'organe respiratoire chez les embryons. Ainsi, lor sque la taille des œufs augmente, la surface respiratoire, rapportée au volu me est diminuée. En conséquence, lorsque la teneur en oxygène de l'eau est la même, les conditions de respiration sont les moins favorables pour les œufs de grande taille. Or, il a été trouvé que les œufs de grande taille renferment davantage de pigments caroténoïdes que les œufs de petite taille (SOIN, 1956).

Cette hypothèse du rôle respiratoire des pigments caroténoïdes a été testée en mesurant la consommation d'oxygène des œufs présentant différents degrés de pigmentation. Aucune corrélation n'a été décelée lors d'incubation dans une eau bien oxygénée. Par contre, une relation directe a été trouvée lors d'incubation dans des eaux pauvres en oxygène. La confirmation de l'intervention des pigments caroténoïdes dans les phénomènes de respiration des cufs a été donnée par JITARIU et al. (1968, 1975).

\subsection{Photoprotection des ceufs}

Le rôle photoprotecteur des pigments caroténoïdes, s'il est bien connu chez les végétaux, n'en est pas moins controversé dans le cas des cuts de poissons. On sait que les œufs de salmonidés sont très sensibles à la lumière, la mortalité pouvant, dans certains cas, avoir lieu quelques heures après exposition aux radiations lumineuses (HAMDORF, 1960). Toutefois, les œufs semblent moins sensibles aux radiations jaunes ou rouges qu'aux radiations bleues ou violettes qui correspondent aux longueurs d'onde des maxima d'absorption des pigments caroténoïdes. Ainsi. HAMDORF (1960) rapporte que des œufs de couleur vive de truite (Salmo irideus) sont plus résistants à l'action de la lumière que des œufs pâles. A l'opposé TORRISSEN et TORRISSEN (1981) notent que des œufs riches 
en astaxanthıne de saumon atlantique (Salmo salar) résistent moins bien que des œufs contenant peu de pigments caroténoïdes.

Mais d'autres facteurs, induits ou non par la présence de lumière, peuvent intervenir et troubler ainsi l'interprétation de tels résultats.

\subsection{Survie des coufs}

Les résultats rapportés par les différents auteurs sont encore contradictoires. SHNAREVICH et SAKHNENKO (1972) notent une forte corrélation négative entre la teneur en caroténoïdes de l'œuf et le taux de survie chez la truite arc-en-ciel (Salmo gairdneri), mais pas chez la truite fario (Salmo trutta). TORRISSEN (1984), quant à lui, ne trouve pas de corrélation entre le taux de pigments caroténoïdes des œufs de saumon (Salmo salar) et le taux de survie.

\subsection{Développement de l'embryon}

La conservation des pigments caroténoïdes dans l'œuf et l'alevin jusqu'à résorption de la vésicule a été étudiée par KITAHARA (1984) chez le saumon (Oncorhynchus keta) et STEVEN (1949) chez la truite fario (Salmo trutta). Il y aurait un transfert progressif des pigments caroténoïdes de la vésicule à l'alevin, sans perte quantitative notable.

Pourtant ce dernier auteur ne croit pas que les pigments caroténoïdes jouent un rôle dans le développement de l'embryon et de la larve. Cette affirmation repose sur les observations suivantes:

- Le transfert de la lutéine et de l'astaxanthine de la vésicule à l'embryon est retardé par rapport à la croissance de cel ui-ci puisqu'à l'éclosion l'embryon qui représente 19 p. 100 du poids de la larve ne renferme que 7 p. 100 des pigments caroténoïdes. tophores.

- Le transfert des caroténoïdes correspond de façon étroite au développement de chroma-

L'hypothèse de l'utilisation exclusive des caroténoïdes pour la pigmentation résulte de travaux consistant à séparer le globule huileux, renfermant 90 p. 100 des caroténoïdes, du reste de la vésicule.

Aucune différence n'a été observée quant à la survie et à la croissance des alevins. Seule la disparition quasi totale des xanthophores et des érythrophores a été notée.

De leur côté GLOVER et al. (1951) ont suivi le métabolisme de l'astaxanthine dans les œufs de saumon(Salmo salar). Pendant les 6 semaines entre la fécondation des œufs et l'éclosion des alevins, aucune modification du taux d'astaxanthine n'intervient. Ainsi à l'éclosion $92 \mathrm{p}$. 100 de l'astaxanthine se retrouve dans le sac embryonnaire et à mesure que l'embryon se développe il y a un transfert constant des caroténoïdes de la vésicule vers l'embryon. Les alevins de 2 mois contiennent encore 80 p. 100 des pigments totaux de l'œuf. Ce transfert ne se fait pas de façon intacte mais s'opère par estérification des pigments sous forme libre (GLOVER et al., 1951; KITAHARA, 1984).

\section{CONCLUSION}

Il peut apparaître, à la lecture de cette revue bibliographique, qu'une grande quantité de documents se rapportent à l'étude de la contribution des pigments caroténoïdes au cycle reproducteur des poissons.

En fait, il n'en est rien et on ne sait que peu de choses sur leur participation directe au processus de la reproduction. Si chez quelques animaux ils apparaissent intimement concernés dans la manifestation de caractères sexuels secondaires (la livrée des mâles par exemple), on les soupçonne de jouer un rôle per se dans la reproduction. Le phénomène marquant demeure cette mobilisation, chez la femelle, des pigments caroténoïdes du muscle vers les gonades. Pour le reste, les autres interventions mentionnées mériteraient d'être approfondies.

\section{BIBLIOGRAPHIE}

ANDRE E., 1926. Influence de l'alimentation sur la pigmentation cutanée des salmonidés. Revue Suisse Zool., 33 (23), 659-669.

CHOUBERT G., 1981. Caroténoïdes et pigmentation. In FONTAINE M. (ed.) Nutrition des poissons, Actes Coll. CNERNA, PARIS Mai 1979, 283-295, CNRS, PARIS.

CROZIER G.F., 1970. Tissue carotenoids in prespawning and spawning sockeye salmon (Oncorhynchus nerka). J. Fish. Res. Bd Can., 27,973-975.

CZECZUGA B., 1974. Carotenoids in the fish milt. Bull. Acad. Pol. Sci. Ser. Sci. Biol., 22, $211-214$

CZECZUGA B., 1975. Carotenoids in fish : IV. Salmonidae and Thymallidae from Polish waters. Hydrobiologia, 36, 223-239. 
CZECZUGA B., 1979a. Carotenoids in fish: XIX. Carotenoids in the eggs of Oncorhynchus keta Walbaum. Hydrobiologia, 63, 45-47.

CZECZUGA B., 1979b. Carotenoids in fish : XX. Carotenoids in Salmo gairdneri Rich. and Salmo Trutta morpha fario L. Hydrobiologia, 64, 251-259.

DEUFEL J., 1965. Pigmentierungs versuche mit canthaxanthin bei Regenbogenforellen. Arch. Fish. Wis., 16, 125-132.

ESCAFFRE A.M., BILLARD R., 1979. Evolution de la fécondabilité des ovules de truite arc-en-ciel Salmo gairdneri laissés dans la cavité abdominale au cours de la période post ovulatoire. Bull. Fr. Piscic., 272, 57-70.

FOX D.L., 1953. Animal biochromes and structural colours. Cambridge Univ. Press, Londres, $380 \mathrm{p}$.

FOX D.L., 1957. The pigments of fishes. In BROWN M.E. (ed.) the physiology of fishes, Vol II, 367-386, Academic Press. New York.

GEORGIEV G.S., 1971. Carotenoids and vitamin A content in Salmo irideus eggs and their significance in the initial periods of the embryogenesis. Folia Balcanica, 2, 1-10.

GILCHRIST B.M., GREEN J., 1960. The pigments of artemia. Proc. Roy. Soc., B 152, 118-136.

GLOVER M., MORTON R.A., ROSEN D.G., 1951. Astaxanthin, cholesterol and lipids in developing salmon eggs. Biochem. J., 50, 425-429.

GOODWIN T.W., 1952. The comparative biochemistry of the carotenoids. Chapman and Hall, Londres, $356 \mathrm{p}$.

HAMDORF K., 1960. Die Beeinflussung der embryonal-und Larvalentwicklung der Regenbogenforelle (Salmo irideus Gibb) durch strahlung in sichtbaren. Bereich. Z. Vgl. Physiol., 42, 525-565.

HARRIS L.E., 1984. Effects of a broodfish diet fortified with canthaxanthin on female fecundity and egg color. Aquaculture 43, 179-183.

HARTMANN M., MEDEM F.G., KUHN R., BIELIG H.J., 1947. Untersuchungen über die Befruchtungsstoffe der Regenbogenforelle. Z. Naturforsch., 2, 330-343.

HAXO F., 1950. Carotenoids of the mushroom Cantarellus cinnabarinus. Botan. Gaz., 112, $228-232$.

HERRING P., 1969. Pigmentation and carotenoid metabolism of the marine isopod/dotea metallica. J. mar. biol. Ass. U.K., 49, 767-779.

HIRAO S., 1967. Carotenoids in fish. Bull. Jap. Soc. Sci. Fish., 33, 853-912.

ISLER O., 1971. Carotenoids. Chemische Reihe band 23, Birkhauser Verlag, Basel 932 p.

JITARIU M., HEFCO H., MULLER W., BOISTEANU I., DAVIDEANU G., 1968. Le rôle des substances caroténoïdes dans le développement embryonnaire des salmonidés. Rev. Roum. Biol. Ser. Zool., $13(2), 117-124$.

JITARIU M., CHERA E., DUCA E., LINCK G., ROTIMBERG P., SZILAGYI I., 1975. Lipido carotenoid metabolism in Salmo gairdneri during embryogenesis. Rev. Roum. Biol. Ser. Biol. Anim., 20(4) 269-274.

KARRER P., JUCKER E., 1950. Carotenoids. Elsevier, New York, 384 p.

KITAHARA T., 1984. Behaviour of carotenoids in the chum salmon (Oncorhynchus keta) during development. Bull. Jap. Soc. Sci. Fish., 50 (3); $531-536$.

KRINSKY N.I., 1971. Carotenoid function. In ISLER O. (ed.) Carotenoids, 669-716, Birkhauser Verlag, Basel.

KUHN R., SÖRENSEN N.A., 1938. Über astaxanthin und ovoverdin. Ber. Stsch. Chem. Ges. 71. 1879-1888.

LEDERER E., 1934. Les caroténoïdes des plantes. Hermann, Paris, 82 p.

LEDERER E., 1935. Les caroténoïdes des animaux. Hermann, Paris, 60 p.

LENEL R., NEGRE-SADARGUES G., CASTILLO R., 1978. Les pigments caroténoïdes chez les crustacés. Arch. Zool. Exp. Gén., 119, 297-334.

MIKIW., YAMAGUCHI K., KONOSUS., 1982. Comparison of carotenoids in the ovaries of marine fish and shellfish. Comp. Biochem. Physiol., 718, 7-11.

MIKI W., YAMAGUCHI K., KONOSU S., WATANABE T., 1984. Metabolism of dietary carotenoids in eggs of red sea bream. Comp. Biochem. Physiol., 71B, 4, 665-668.

MIKULIN A.Y., SOIN S.G., 1975. The functional significance of carotenoids in the embryonic development of Teleost. J. Ichthyol., 15 (5), 749-759.

MORRISON J.K., SMITH C.E., 1981. The effects of dietary carotenoid supplementation on egg quality of brook trout. Inf. Leafl. 19, Fish. Dev. Centre, Bozeman, MT 6 pp.

QUANTZ G., 1980. Über den Einfluss von carotinoidreichem Trocken futter auf die Eibefruchtung der Regenbogenforelle (Salmo gairdneri R.). Arch. Fischwiss, 31 (1), 29-40. 
SIMPSON K.L., KATAYAMA T., CHICHESTER C. O., 1981. Carotenoids in fish feeds. In BAUERNFEIND J.C. (ed.) carotenoids as colorants and vitamin A precursors. 463-538, Academic Press, New-York.

SHNAREVICH I.D., SAKHNENKO E.G., 1971. Dynamics of carotenoids in tissue and organ of fish relative to the sexual cycle (russe). Gidrobiol. $2 h ., 7,90-93$.

SHNAREVICH J.D., SAKHNENKO E.G., 1972. On the influence of carotenoids content in the egg of trout on development of its young (russe). Nautch. Dokl. Vysch. Shk. Ser:Biol. Nauki. 12, 20-23.

SOIN S.G., 1956. On the respiratory role of the spawn carotenoid pigment of the salmonids. Vopr. Ichthyol., 35 (9). 1362-1369.

SOIN S.G., 1962. Embryonic respiratory adaptations in fishes and characteristics of their development in Baikalian sculpins. Vopr. Ichthyol., 2 (2), 13-16.

STEVEN D.M., 1948. Studies on animal carotenoids. I. Carotenoids of the brown trout (Salmo trutta Linn.). J. Exp. Biol., 25, 369-387.

STEVEN D.M., 1949. Studies on animal carotenoids. II. Carotenoids in the reproductive cycle of the brown trout. J. Exp. Biol., 26, 295-303.

TAKEUCHIK., 1960. The behavior of carotenoid and distribution of xanthophores during development of the medaka (Oryzias latipes). Embryologia, 5 (2), 170-177

TORRISSEN O.J., 1984. Pigmentation of salmonids. Effect of carotenoids in eggs and start feeding diet on survival and growth rate. Aquaculture, 43, 185-193.

TORRISSEN O., TORRISSEN K.R., 1981. The effects of lights on the mortality of different pigmented Atlantic salmon (Salmo salar) eggs. Intern. Counc. Expl. Sea. CM/M: 16, 8 pp.

TSUKUDA N., AMANO K., 1966. Studies on the discoloration of red fishes. I. Content of carotenoid pigments in eighteen species of red fishes. Bull. Jap. Soc. Sci. Fish., 32, 334-345.

ZECHMEISTER L., 1962. Cis-trans isomeric carotenoids, vitamins A and arylpolyenes. Springer Verlag, Vienne, $215 \mathrm{p}$. 